\title{
Provendo Abstrações de Alto Nível para GPUs na SPar
}

\author{
Dinei André Rockenbach ${ }^{1}$, Dalvan Griebler ${ }^{1}$, Luiz Gustavo Fernandes ${ }^{1}$ \\ ${ }^{1}$ Escola Politécnica, Grupo de Modelagem de Aplicações Paralelas (GMAP), \\ Pontifícia Universidade Católica do Rio Grande do Sul (PUCRS), Porto Alegre, Brasil \\ dinei.rockenbach@edu.pucrs.br, dalvan.grieblerepucrs.br
}

\begin{abstract}
Resumo. O presente trabalho apresenta uma extensão à linguagem SPar para suportar o paralelismo heterogêneo combinado de CPU e GPU através de anotações $C++11$ em aplicações de processamento de stream. Os testes sugerem melhoras significativas de desempenho com poucas modificações no código.
\end{abstract}

\section{Estudo e Pesquisa}

Os novos hardwares surgidos nos últimos anos têm seguido a tendência crescente no volume de dados gerados pelo uso das tecnologias digitais. Na vanguarda da busca por mais desempenho estão as Graphics Processing Units (GPUs), hardwares massivamente paralelos originalmente desenhados para processamento gráfico, mas que hoje em dia são largamente utilizados em várias tarefas de cunho geral. O advento das GPUs impulsionou aplicações como carros com direção autônoma, ray tracing em tempo real, aprendizado profundo na inteligência artificial e realidade virtual (VR). Porém, esse ambiente heterogêneo com GPUs e Central Processing Units (CPUs) paralelas apresenta um desafio adicional para o desenvolvimento de software paralelo.

A programação estruturada é uma alternativa para facilitar o desenvolvimento dessas aplicações através do uso de padrões paralelos. Estes padrões simplificam a programação utilizando estruturas algorítmicas (algorithmic skeletons) com fluxos de execução paralelos pré-programados. Porém, para a exploração de paralelismo em GPU atualmente tanto a academia quanto a indústria usam CUDA e OpenCL, que não oferecem interfaces de programação estruturada e cujos desenvolvedores precisam lidar com conceitos de arquitetura de hardware de baixo nível. Existe uma carência de abstrações de programação paralela de alto nível que lidam com o paralelismo de CPU e GPU combinados.

Muitas aplicações que desejam realizar processamento na GPU são aplicações de processamento de stream, que computam uma sequência de operadores sobre dados que são gerados em fluxo contínuo. Cada um dos operadores pode processar diferentes dados simultaneamente, que é o paralelismo de stream. Esse paralelismo é adequado para a CPU, enquanto que o paralelismo de dados é adequado para processamento na GPU. Por exemplo, uma aplicação de geração de vídeo expõe paralelismo de stream na sequência de imagens a ser processada, porém cada imagem expõe paralelismo de dados uma vez que a cor de cada pixel pode ser computada individualmente. Este trabalho continua a proposta apresentada em [Rockenbach et al. 2019] para fornecer abstrações de alto nível para a exploração do paralelismo em ambientes heterogêneos com CPUs e GPUs.

A SPar [Griebler et al. 2017] é uma linguagem de domínio específico (DomainSpecific Language ou DSL) focada no paralelismo de stream. Ela oferece cinco atributos $\mathrm{C}++11$ que podem ser inseridos no código através de anotações. O código anotado é processado por um compilador que efetua transformações source-to-source, inserindo chamadas a bibliotecas de nível mais baixo, tais como FastFlow, TBB e OpenMP, para 
explorar o paralelismo de stream através da geração eficiente dos padrões pipeline e farm, ou a composição de pipeline com farm(s). Para permitir a exploração do paralelismo massivo das GPUs utilizando as abstrações de alto nível da SPar, foi necessário estender a linguagem incluindo três novos atributos: (1) Pure, para indicar estágios ou blocos de código puros que podem ser enviados para a GPU; (2) Batch, para diminuir a granularidade dos elementos do stream e fornecer maior volume de dados para cada chamada à GPU; e (3) Reduce, para indicar operações de reduções dentro de blocos de código puro.

Para suportar estes novos atributos, foram elaboradas regras de transformação source-to-source, que foram implementadas no compilador a fim de gerar os padrões paralelos map e map-reduce utilizando a biblioteca GSParLib [Rockenbach 2020]. Essa biblioteca foi desenvolvida com uma interface de programação estruturada unificada e um ambiente de execução agnóstico ao driver da plataforma de hardware. Ela oferece os padrões paralelos map e reduce sobre os drivers CUDA e OpenCL.

Os testes de desempenho da aplicação Lane Detection foram executados sobre 3.169 frames com resolução $1242 \times 375$ do conjunto de dados KITTI e foram executados em uma máquina com CPU Intel® Core $^{\mathrm{TM}}$ I9-7900X @ $3.3 \mathrm{GHz}$ e uma GPU Titan Xp com $12 \mathrm{~GB}$ GDDR5X@2400 MHz de memória. Partindo do código sequencial, foram necessárias apenas três anotações da SPar para explorar o paralelismo de CPU e outras três anotações para explorar o paralelismo heterogêneo combinado de CPU e GPU. Na Figura 1 é possível observar que a SPar apresentou melhoras de desempenho significativas, com $7.9 \times$ speedup e 255 FPS na versão multi-core (em verde) com nove workers, $11.5 \times$ speedup e 374 FPS na versão GPU com OpenCL (em laranja) e $10.7 \times$ speedup e 348 FPS na versão GPU com

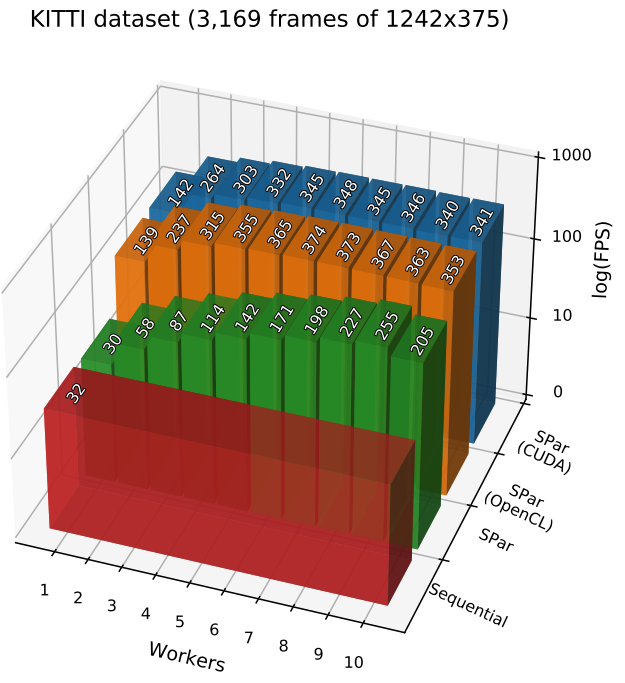

Figura 1. Throughput do Lane Detection. CUDA (em azul). Pode-se notar que quando há mais de 5 workers nas versões que utilizam a GPU, o gargalo da aplicação passa a ser esse processador, uma vez que os testes foram realizados com uma única GPU.

Com essa extensão, a linguagem SPar oferece suporte ao paralelismo de dados em GPU e paralelismo de stream em CPU, permitindo explorar eficientemente o paralelismo heterogêneo em aplicações de processamento de stream com abstrações de alto nível.

\section{Referências}

Griebler, D., Danelutto, M., Torquati, M., and Fernandes, L. G. (2017). SPar: A DSL for High-Level and Productive Stream Parallelism. Parallel Processing Letters, 27(01):1740005.

Rockenbach, D. A. (2020). High-Level Programming Abstractions for Stream Parallelism on GPUs. Master's thesis, School of Technology - PPGCC - PUCRS, Porto Alegre, Brazil.

Rockenbach, D. A., Griebler, D., and Fernandes, L. G. (2019). Proposta de Suporte ao Paralelismo de GPU na SPar. In Escola Regional de Alto Desempenho (ERAD-RS), page 4, Três de Maio, BR. Sociedade Brasileira de Computação (SBC). 